\title{
Processing of empty and filled time intervals in pigeons
}

\author{
DOUGLAS S. GRANT and DIANE C. TALARICO \\ University of Alberta, Edmonton, Alberta, Canada
}

\begin{abstract}
Pigeons were trained initially with 2- and 8-sec empty or filled intervals as sample stimuli. Interval onset and termination was signaled by 1-sec start and stop markers. Following retention and psychophysical testing, both groups were trained with the alternative type of interval, and the tests were repeated. Group empty-first demonstrated a choose-long effect with both empty and filled intervals. Group filled-first demonstrated a weak (and nonsignificant) choose-short effect with filled intervals and a robust choose-long effect with empty intervals. Both groups tended to time the markers and to add that duration to the sample duration only on filled-interval trials. Initial training with empty intervals alters the way pigeons process temporal information on filled-interval trials, whereas initial training with filled intervals has little effect on the processing of temporal information on empty-interval trials.
\end{abstract}

In many studies, a symbolic matching-to-sample task has been used to study memory for event duration in pigeons (see Grant, Spetch, \& Kelly, 1997, for a review). In a typical experiment, trials begin with presentation of a houselight or a keylight stimulus for either a short (e.g., 2 -sec) or a long (e.g., 8-sec) duration. Following termination of the duration sample, two comparison stimuli are presented for a choice. Choice of one comparison is reinforced on trials initiated by the short sample, and choice of the alternative comparison is reinforced on trials initiated by the long sample.

When subsequently tested at delays longer than the training delay, pigeons demonstrate a choose-short effect (CSE; e.g., Grant \& Kelly, 1996, 1998; Grant \& Spetch, 1991, 1993, 1994; Kraemer, Mazmanian, \& Roberts, 1985; Santi, Bridson, \& Ducharme, 1993; Spetch, 1987; Spetch \& Rusak, 1992a; Spetch \& Wilkie, 1982, 1983; Wilkie, 1987). That is, when tested at delays longer than the training delay, pigeons tend to choose the comparison associated with the short sample. The dominant explanation maintains that pigeons code durations in a retrospective, analogical form and that the CSE arises because the analogical representation foreshortens during a delay interval (e.g., Grant, 1993; Grant \& Spetch, 1991, 1993; Grant et al., 1997; Roberts, Macuda, \& Brodbeck, 1995; Santi et al., 1993; Spetch, Grant, \& Kelly, 1996; Spetch \& Rusak, 1992b; Spetch \& Wilkie, 1982, 1983).

Recently, the study of memory for time intervals in pigeons has been extended to the case of empty time intervals (Grant, 2001; Santi, Hornyak, \& Miki, 2003; Santi,

This research was supported by Grant OGP 0443 from the Natural Sciences and Engineering Research Council of Canada. Correspondence concerning this article may be directed to D. S. Grant, Department of Psychology, University of Alberta, Edmonton, AB, T6G 2E9 Canada (e-mail: douglas.grant@ualberta.ca).
Ross, Coppa, \& Coyle, 1999). In this procedure, intervals to be timed are bounded by brief start and stop markers. Grant (2001), for example, used a 1-sec presentation of three red lights as the start marker and a 1-sec presentation of three green keylights as the stop marker for some pigeons. In all three studies, the empty intervals to be timed were $2 \mathrm{sec}$ (short) and $8 \mathrm{sec}$ (long) in duration. When memory for the empty interval was assessed at delays considerably longer than that employed during training, all three studies revealed a choose-long effect (CLE). Hence, in contrast to the typical result obtained when filled intervals are employed, the empty-interval procedure results in a tendency to choose the comparison associated with the long, rather than the short, sample at longer delays.

The primary purpose of the experiments reported in this article was to compare pigeons' performance with empty and filled intervals within the same experiment. In an article that appeared after data collection for the present experiments had been completed, Santi et al. (2003) also studied memory for empty and filled intervals in the same experiment. In that study, a within-subjects design was used in which 2- and 8-sec filled intervals were followed by one set of comparisons and 2- and 8-sec empty intervals were followed by the alternative set of comparisons. When tested at delays considerably longer than the 0 -sec baseline delay, the retention functions for empty intervals revealed a CLE, and those for filled intervals revealed a CSE.

The design of the experiments reported in this article allowed for both between- and within-subjects comparison of performance with empty and filled time intervals. In particular, two groups of pigeons were trained initially with only one set of intervals, either empty (group emptyfirst) or filled (group filled-first), and received retention and psychophysical testing. Subsequently, both groups were trained and tested with the alternative set of intervals mapped to new comparisons, while training contin- 
ued on the initially learned task. The retention and psychophysical tests were then repeated, with both groups now being tested with both empty and filled intervals.

\section{EXPERIMENT 1}

Pigeons were trained to respond to one comparison after a short $(2$-sec) interval and to the alternative comparison after a long $(8-\mathrm{sec})$ interval. In both groups, onset of an interval was marked by presentation of keylights illuminated with a white circle, and termination of the interval was marked by keylights illuminated with yellow light. In group empty-first, the interval between termination of the start marker and onset of the stop marker was spent in complete darkness. In group filledfirst, this interval was spent in the presence of a keylight illuminated by a white field containing a black dot in the center. As in Grant's (2001) experiments, training trials involved a variable delay in the range of $1-3 \mathrm{sec}$. The purpose of this aspect of the procedure was to reduce the likelihood that onset of the comparisons, rather than onset of the second marker, would terminate timing.

Following acquisition, the pigeons received consecutive retention tests in which the delays were $0.5,2( \pm 1)$, and $10 \mathrm{sec}$ in the first test, and were $2( \pm 1), 10$, and $20 \mathrm{sec}$ in the second test. Because the procedure in group empty-first was highly similar to that employed by Grant (2001) and Santi et al. (1999), a CLE at delays longer than the training delay was anticipated. Because the procedure in group filled-first was highly similar to that employed in numerous experiments demonstrating a CSE (except, of course, for the use of start and stop markers), a CSE was anticipated in this group. Santi et al.'s (2003) recent within-subjects comparison also leads to the expectation of a CLE with empty intervals and a CSE with filled intervals.

\section{Method}

\section{Subjects}

Sixteen naive Silver King pigeons (Columba livia), each approximately 12 months old at the start of training, were reduced to and maintained at $80 \%$ of their free-feeding weight. The birds were housed individually in wire-mesh cages and were given unrestricted access to water and health grit. Home cages were kept in a colony room, illuminated on a 12:12-h light:dark cycle with light onset at $0600 \mathrm{~h}$. Eight birds were assigned at random to group empty-first, and the remaining 8 were assigned to group filled-first.

\section{Apparatus}

Training and testing was conducted in eight identical operant chambers, each measuring $29.0 \times 29.0 \times 24.0 \mathrm{~cm}$ (height $\times$ length $\times$ width). In each chamber, a horizontal alignment of three circular pecking keys (each $2.5 \mathrm{~cm}$ in diameter, and with side keys separated edge-to-edge from the center key by $3.0 \mathrm{~cm}$ ) was centered along one end wall. The key alignment was raised $22.5 \mathrm{~cm}$ from the barred-floor base of the chamber. Affixed behind each key was an Industrial Electronics, Inc. (Van Nuys, CA) in-line projector. All three projectors were capable of transilluminating red, green, and yellow fields, a white line oriented either vertically or horizontally, a white circle on a black background, and a black dot on an otherwise white key. A force greater than $0.15 \mathrm{~N}$ applied to any key was recorded as a keypeck.
}

The top edge of a $5.0 \mathrm{~cm}$ wide $\times 5.5 \mathrm{~cm}$ high rectangular opening was located $9.0 \mathrm{~cm}$ directly beneath the edge of the middle key. This opening provided access to a retractable food magazine. A $28-\mathrm{V}$ lamp, recessed within the magazine opening, was activated when the food magazine was raised. Each chamber was enclosed in a sound- and light-attenuating booth. Within each booth, an exhaust fan provided ventilation and, supplemented by an external white noise generator, masking auditory stimulation. All the experimental booths were isolated in the same darkened running room. The only illumination inside the chamber was provided by activation of keylights and the magazine light. Experimental events were controlled from, and responses were recorded by, a microcomputer located in an adjoining room. Experimental sessions were conducted daily, 6 days per week, and began at approximately the same time each day.

\section{Procedure}

Preliminary training. The birds were initially trained to eat grain from the raised and illuminated magazine. Once the birds were eating reliably, they were autoshaped to peck red and green or horizontal and vertical, depending on which dimension was scheduled to serve as the comparisons for that bird. Once all the birds were pecking reliably, they were advanced to training.

Training. For the birds in both groups, each trial began with a 1 -sec presentation of a white circle on all three pecking keys (start marker). Termination of the circles was followed by a dark interval of either $2 \mathrm{sec}$ (short) or $8 \mathrm{sec}$ (long) in group empty-first and was followed by intervals of equivalent duration that were spent in the presence of a black dot on a white background in group filled-first. The end of the interval was marked, in both groups, by onset of yellow light on all three pecking keys that lasted for $1 \mathrm{sec}$ (stop marker). Termination of the yellow keylights was followed by a short, variable delay that ranged from 1 to $3 \mathrm{sec}$ in 0.5 -sec increments.

For 4 birds in each group, termination of the delay was followed immediately by presentation of the red comparison stimulus on one side key and the green comparison stimulus on the remaining side key. A single peck to either comparison stimulus terminated both. For 2 subjects in each group, a peck to the red comparison stimulus after the short sample and a peck to the green comparison stimulus after the long sample were designated as correct matches and thus resulted in reinforcement, consisting of $2.5 \mathrm{sec}$ of magazine-illuminated access to mixed grain from the food hopper. Alternatively, a peck to green after a short sample and a peck to red after a long sample were designated as incorrect matches and thus resulted in nonreinforcement, consisting of $2.5 \mathrm{sec}$ of darkness without mixed grain. For 2 other subjects in each group, these contingencies were reversed.

For the remaining 4 birds in each group, termination of the delay was followed immediately by presentation of the horizontal line comparison stimulus on one side key and the vertical line comparison stimulus on the remaining side key. A single peck to either comparison stimulus terminated both. For 2 subjects in each group, a peck to the horizontal comparison stimulus after the short sample and a peck to the vertical comparison stimulus after the long sample were designated as correct matches and thus resulted in reinforcement. Alternatively, a peck to vertical after a short sample and a peck to horizontal after a long sample were designated as incorrect matches and thus resulted in nonreinforcement. For 2 other subjects in each group, these contingencies were reversed.

Sessions consisted of an equal number of short- and long-sample trials. Within each session, the side key location of the correct comparison stimulus was varied randomly across trials, with the restriction that, over all trials with each sample type, the correct comparison stimulus appeared equally often on the left and the right keys.

Each trial concluded with the onset of an intertrial interval (ITI), the duration of which varied randomly in 5-sec increments within a range of 10 to $30 \mathrm{sec}(M=20 \mathrm{sec})$. Each session consisted of 64 trials. All the pigeons received 96 sessions of training. At that point, with one exception in each group, all the birds were consistently 
above $85 \%$ correct. The 2 birds that failed to reach this level of accuracy were dropped from the experiment; hence, $n=7$ in each group.

Extended-delay testing. Testing consisted of two consecutive test phases, each consisting of eight test sessions. Each test session after the first was preceded by two baseline sessions identical to those in training. In both test phases, all the parameters and procedures for the test sessions were identical to those for training, except that the delay between termination of the second marker and onset of comparison stimuli varied across three values. In the first test phase, on a randomly determined $75 \%$ of the trials, the delay was equivalent to that in training and, hence, varied between 1 and $3 \mathrm{sec}$ in 0.5 -sec increments. On a randomly chosen half of the remaining $25 \%$ of the trials, a 0.5 -sec delay was interpolated between sample termination and comparison stimuli onset. On the remaining half of these trials, a 10 -sec delay occurred. In the second test phase, as in the first, on a randomly determined $75 \%$ of the trials, the delay was equivalent to that in training and, hence, varied between 1 and $3 \mathrm{sec}$ in 0.5 -sec increments. On a randomly chosen half of the remaining $25 \%$ of the trials, a $10-\mathrm{sec}$ delay was interpolated between sample termination and comparison stimuli onset. On the remaining half of these trials, a 20 -sec delay occurred.

In both test phases, both samples occurred equally often before each of the three delays, and the position of the correct comparison stimulus was balanced within sample type and delay interval factors. Correct responses on extended-delay trials were reinforced in accord with the contingencies of training.

In all the experiments reported in this article, statistical analyses employed $p<.05$ to define the critical region for rejection of the null hypothesis.

\section{Results \\ Training}

Figure 1 shows accuracy in both groups on short- and long-sample trials as a function of blocks. Acquisition of accurate matching on long-sample trials in group filled-first lagged somewhat behind that with the other sample types. A group $\times$ sample duration $\times$ block analysis of variance (ANOVA) revealed a significant main effect of block $[F(23,276)=41.22]$ and a significant triple interaction $[F(23,276)=1.84]$, reflecting the somewhat slower acquisition on long trials in group filled-first.

\section{Extended-Delay Testing}

The results of the first phase of testing, in which the delays were $0.5,2 \pm 1$ (as in training), and $10 \mathrm{sec}$, are shown in Figure 2. As was anticipated, group empty-first (top panel) demonstrated a strong tendency to choose the long-associated comparison stimulus at delays longer than the training delay, a CLE. In contrast to expectation, however, there was little evidence of a CSE in group filled-first (bottom panel).

A group $\times$ sample duration $\times$ delay ANOVA revealed a significant three-way interaction $[F(2,24)=12.93]$. Hence, separate sample duration $\times$ delay ANOVAs were conducted on the data from each group. In group emptyfirst, the sample duration $\times$ delay interaction was significant $[F(2,12)=16.81]$, revealing a reliable CLE. However, in group filled-first, the sample duration $X$ delay interaction was not significant $(F<1)$, revealing no reliable retention asymmetries.

The results of the second phase of testing, in which the delays were $2 \pm 1,10$, and $20 \mathrm{sec}$, are shown in Figure 3 . The results of the second test were highly similar to those of the first, in that group empty-first (top panel) demonstrated a robust CLE, whereas retention asymmetries were not apparent in group filled-first (bottom panel). The data were analyzed in the same manner as those from the first test, and again, the three-way interaction was significant $[F(2,24)=4.34]$. Follow-up

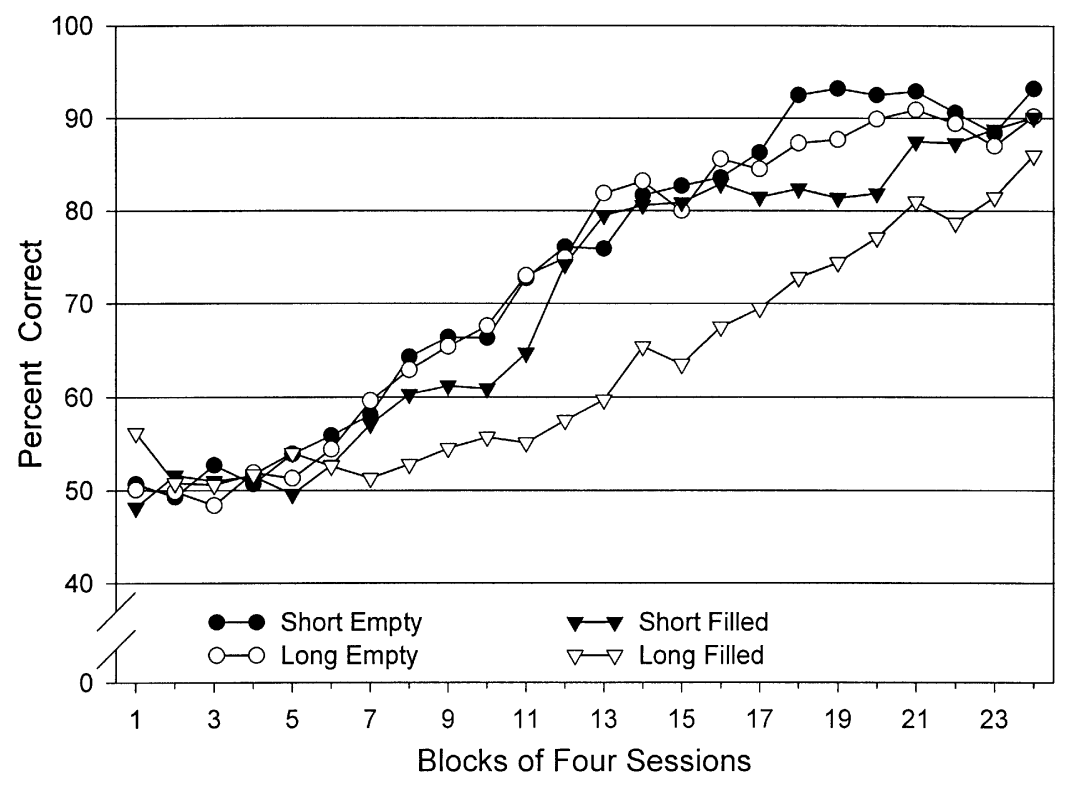

Figure 1. Percentages of correct responses on short-empty and long-empty trials in group empty-first and on short-filled and long-filled trials in group filled-first as a function of blocks of four sessions. Data from the 2 birds, 1 in each group, that failed to reached criterion are not included. 

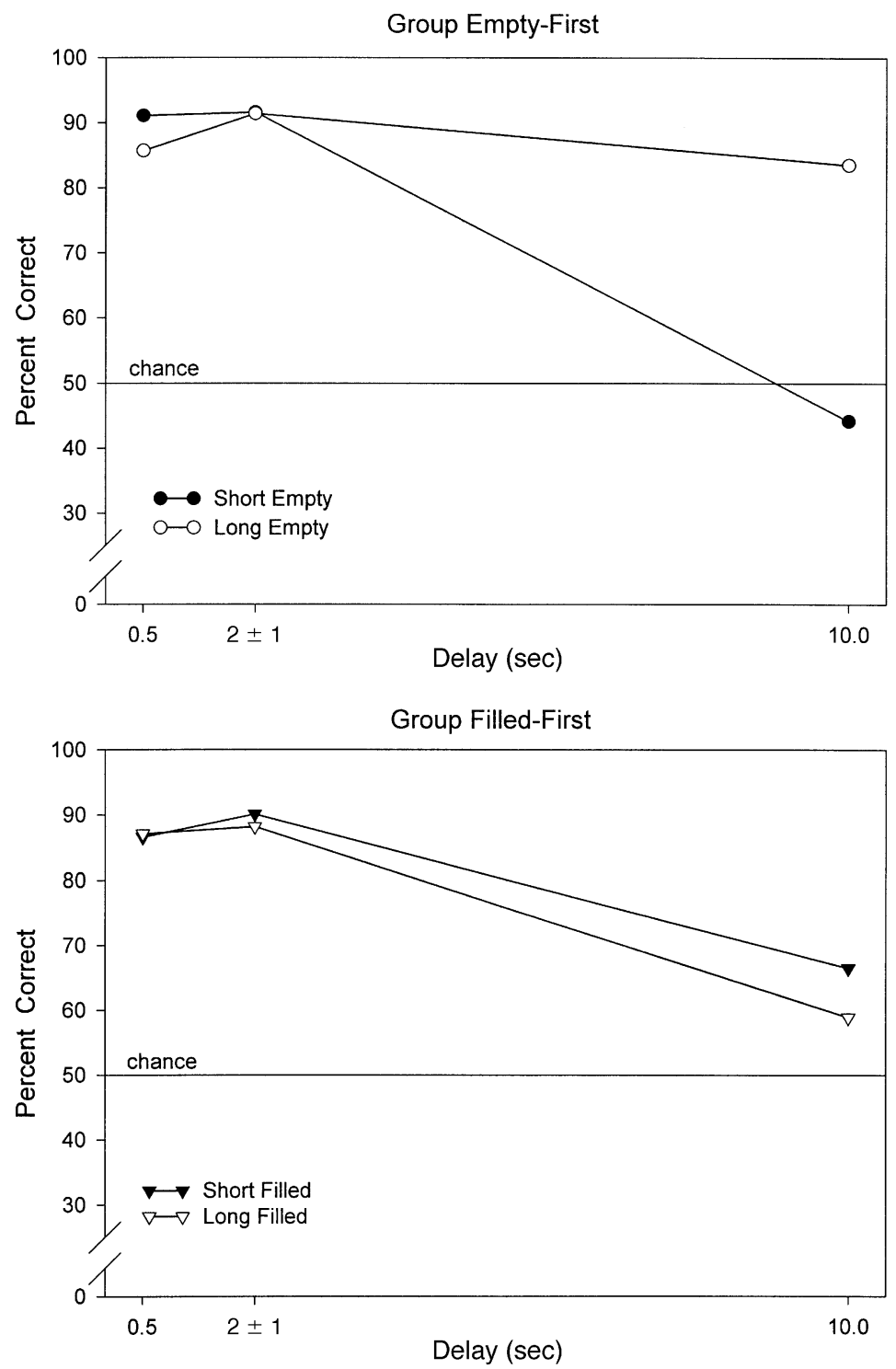

Figure 2. Percentages of correct responses as a function of delay on shortand long-sample trials in groups empty-first (top graph) and filled-first (bottom graph) in the first delay test in Experiment 1.

ANOVAs revealed a significant CLE in group emptyfirst $[F(2,12)=4.63]$ and the lack of reliable retention asymmetries in group filled-first $(F<1)$.

\section{Discussion}

In accord with the results of Grant (2001) and Santi et al. (1999; Santi et al., 2003), both extended-delay tests revealed a robust CLE at delays longer than the training delay in birds trained and tested with empty intervals. However, in contrast to expectation, the pigeons trained with filled intervals did not demonstrate a CSE, and instead, accuracy declined toward chance level at about the same rate on both short- and long-sample trials. It is important to note that although group filled-first did not demonstrate a CSE, neither did it demonstrate a CLE.
This finding suggests that the CLE in group empty-first resulted from the use of empty intervals, rather than from the use of start and stop markers.

The failure to obtain a reliable CSE with filled intervals is surprising, particularly in light of Santi et al.'s (2003) within-subjects test that revealed a CLE with empty intervals and a CSE with filled intervals. Several procedural differences between filled-interval trials in the present experiment and those in Santi et al.'s (2003) experiment can be identified. First, we used a variable delay during training that varied in length between 1 and 3 sec. However, Grant and Kelly (1998) used an identical variable-delay training procedure with 2 - and 8 -sec filled intervals and obtained a reliable CSE at 15- and 30-sec delays. Moreover, Spetch and Rusak (1992a) used 

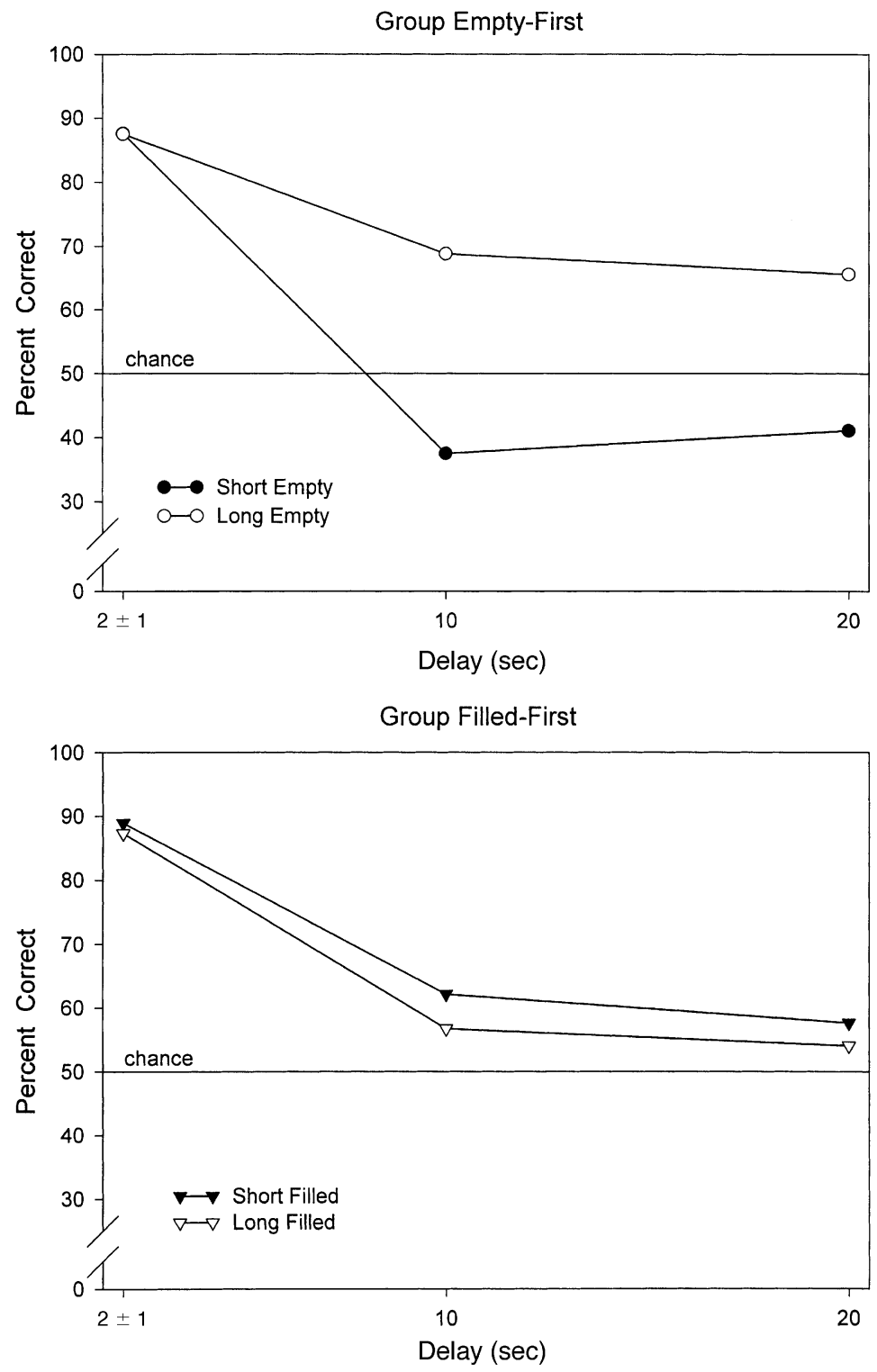

Figure 3. Percentages of correct responses as a function of delay on shortand long-sample trials in groups empty-first (top graph) and filled-first (bottom graph) in the second delay test in Experiment 1.

delays of $2,4,6$, and $8 \mathrm{sec}$ during training with 2 - and 8 -sec food samples and found a CSE at a 20 -sec delay. In contrast to these results, Dorrance, Kaiser, and Zentall (2000, Experiment 1, Group Dark-Dark) found parallel retention functions with 2 - and 10 -sec filled intervals during testing with delays ranging from 0 to $16 \mathrm{sec}$ following training with delays of $0,1,2$, and $4 \mathrm{sec}$. At present, therefore, the literature on the role of variable-delay training on retention asymmetries with filled intervals is somewhat ambiguous.

A second procedural difference on filled-interval trials is that Santi et al. (2003) did not present start and stop markers, whereas markers were presented on both emptyand filled-interval trials in the present experiment. How- ever, we have often used a preparatory stimulus to signal the onset of filled intervals, and we have routinely obtained a CSE with that procedure (e.g., Grant \& Kelly, $1996,1998,2001)$. These preparatory stimuli differ from the start marker used in the present experiment in that the preparatory stimulus could be terminated by a keypeck or by the passage of $5 \mathrm{sec}$ in the absence of a keypeck, whereas the start marker was always $1 \mathrm{sec}$ in duration in the present experiment.

A reviewer of an earlier version of this article asked whether the absence of a reliable CSE in group filledfirst might have occurred as a function of averaging across birds, some of which demonstrated a robust CSE and others of which demonstrated a robust CLE. Inspec- 
tion of the data from both delay tests suggested that that was not the case. To provide a more definitive assessment of this possibility, data from group filled-first in both delay tests were subjected to a second sample duration $\times$ delay ANOVA. However, in these analyses, only data from the birds that demonstrated higher accuracy on short-sample than on long-sample trials at delays longer than baseline were included (i.e., birds demonstrating a choose-short tendency). Data from 4 birds satisfied this condition in the first delay test, and the data from 6 did so in the second delay test. The sample duration $X$ delay interaction was not significant in either analysis $[F(2,6)=$ 3.94 in the first delay test and $F(2,10)=2.01$ in the second delay test]. Thus, a statistically reliable CSE was not obtained even when data from only those birds whose accuracy was consistent with a CSE were included in the analyses.

Hence, the pattern of decline in accuracy as a function of delay in group filled-first suggests that the pigeons in this group were employing some form of categorical (e.g., short/long or red/green), rather than analogical, code. This issue will be considered further in the General Discussion section.

Finally, note that acquisition on long-sample trials lagged somewhat behind that on short-sample trials in group filled-first, whereas acquisition proceeded at an approximately equivalent rate on short- and long-sample trials in group empty-first. Differential rates of acquisition with short- and long-filled samples has not been routinely observed in prior research in our laboratory, and its significance in the present experiment is unclear.

\section{EXPERIMENT 2}

Pigeons received probe trials in which the sample duration varied between 2 and $8 \mathrm{sec}$ in 1 -sec increments. This type of test has been used frequently to determine the point of subjective equality (PSE). The PSE is the sample duration at which pigeons are indifferent between short and long and are equally likely to respond short as to respond long to that duration. A number of studies using filled intervals have revealed that the PSE falls at the geometric mean between the short and the long samples (e.g., Gibbon, 1986; Miki \& Santi, 2001; Spetch \& Wilkie, 1983; Stubbs, 1968). The geometric mean is computed as the square root of the product of the short and the long durations. Hence, it was anticipated that the PSE would be at or close to $4 \mathrm{sec}$ in group filled-first. To date, the PSE has not been assessed with empty intervals and pigeon subjects.

On any particular probe session, the duration of the start and stop markers on probe trials was $0.5,1$ (as in baseline), or $2 \mathrm{sec}$. The purpose of this manipulation was to determine whether either group was timing the markers and combining that duration with that experienced during the sample. If so, then with markers of $0.5,1$, and $2 \mathrm{sec}$, the functional duration of the nominal 2 -sec sample would be 3,4 , and $6 \mathrm{sec}$, and the functional duration of the nominal 8 -sec sample would be 9,10 , and $12 \mathrm{sec}$.
Hence, evidence of timing the markers and combining that duration with that experienced during the sample would be provided if the proportion of long choices increased and, hence, the PSE decreased as the duration of the start and stop markers increased.

Santi et al. (2003) manipulated the duration of the second marker on empty-interval trials. As the duration of the marker increased from $500 \mathrm{msec}$ (baseline) to 750 and $1,000 \mathrm{msec}$, accuracy decreased slightly although, importantly, accuracy on short- and long-sample trials was not affected differentially. This finding suggests that behavior on empty-interval trials was not under the control of the remembered durations of the markers. Hence, we anticipated that the percentage of long choices would not increase and, hence, that the PSE would not decrease as marker duration increased from 0.5 to $2 \mathrm{sec}$ on emptyinterval trials.

\section{Method}

\section{Subjects and Apparatus}

The 14 subjects ( 7 in each group) that met criterion in Experiment 1 participated. The apparatus was the same as that in Experiment 1.

\section{Procedure}

Twenty baseline sessions, identical to the training sessions in Experiment 1, intervened between the end of Experiment 1 and an initial psychophysical test that is not reported in this article. That test was identical to the present test, except that the markers were always $1 \mathrm{sec}$ in duration and the experiment consisted of 16 , rather than 48 , sessions. Twelve baseline sessions intervened between the end of that test and the onset of the present experiment.

Sessions consisted of 55 trials, 48 of which were identical to the training trials employed in Experiment 1 . The remaining 7 trials were probe trials on which the interval to be timed was $2,3,4,5,6$, 7 , or $8 \mathrm{sec}$ in duration. Within each session, Trials 7, 15, 23, 31, 39, 47 , and 55 were probe trials, and each of the seven probe durations was presented on 1 probe trial. The delay on all probe trials was $2 \mathrm{sec}$, the mean of the variable training delay. The position of the correct comparison stimulus on probe trials was determined randomly, and a response to either comparison stimulus was reinforced on probe trials.

There were three types of probe sessions that differed in terms of the length of the start and stop markers on probe trials: $0.5,1$, or $2 \mathrm{sec}$. Each of the three marker durations was employed once in every 3 probe sessions, and the order in which the three marker durations were tested was determined randomly for each set of 3 probe sessions. The experiment consisted of 48 consecutive probe sessions, 16 with each of the three marker durations.

In determining the PSE, SigmaPlot 8.0 was used to find the bestfitting parameters of the linear regression equation at each of the three marker durations. Because of a ceiling effect on the percentage of long choices at probe durations of 7 and $8 \mathrm{sec}$, the linear regression was performed on data from probe trials in which the sample duration was between 2 and 6 sec. Hence, the regression was performed using five probe durations - one at the hypothesized PSE (i.e., $4 \mathrm{sec}$ ), two below the hypothesized PSE (i.e., 2 and $3 \mathrm{sec}$ ), and two above the hypothesized PSE (i.e., 5 and $6 \mathrm{sec}$ ). The regression equation and best-fitting parameters were used to solve for the PSE, the calculated duration at which the pigeons would be indifferent between the comparisons (i.e., $50 \%$ long choices).

\section{Results}

Figure 4 shows the percentage of long choices as a function of sample duration on probe trials, with marker 
duration as the parameter. The data from group emptyfirst are shown in the upper graph, and those from group filled-first are shown in the lower graph. Not surprisingly, both groups demonstrated a strong relation between sample duration and the percentage of long choices, with the percentage of long choices being about $10 \%$ at the 2 -sec sample duration and rising to about $90 \%$ at the 8 -sec sample duration. In group empty-first, there was little evidence of a systematic relation between marker duration and percentage of long choices. In group filled-first, in contrast, the percentage of long choices increased as a function of increases in marker duration.
A group $\times$ sample duration $\times$ marker duration ANOVA was performed on the data from probe trials. Because that ANOVA revealed a significant marker duration $\times$ group interaction $[F(2,24)=4.10]$, separate sample duration $\times$ marker duration ANOVAs were performed on the data for each group. In group empty-first, the only significant term was the main effect of sample duration $[F(6,36)=62.62]$. In group filled-first, both main effects were significant [sample duration, $F(6,36)=$ 137.39; marker duration, $F(2,12)=10.78]$.

Mean PSEs in group empty-first were 4.02, 3.81, and 3.80 with $0.5-, 1-$, and 2 -sec marker durations, respec-
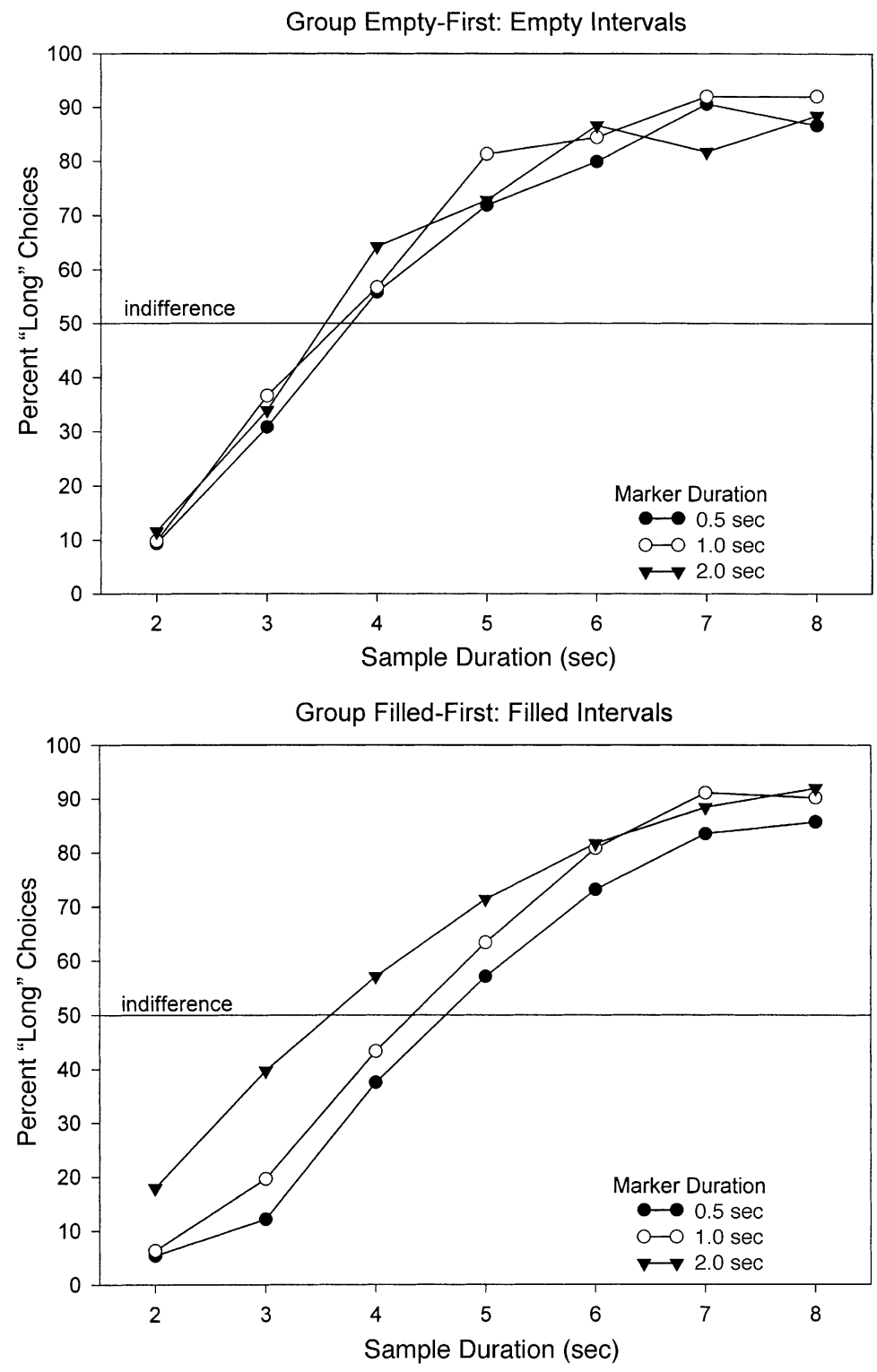

Figure 4. Percentages of choices of the long-associated comparison stimulus as a function of sample stimulus duration in groups empty-first (top graph) and filled-first (bottom graph) in Experiment 2. Marker duration (i.e., duration of the start and stop signals) is the parameter. 
tively. In group filled-first, mean PSEs were 4.71, 4.37, and 3.78 with $0.5-, 1-$, and 2-sec marker durations, respectively. A group $\times$ marker duration ANOVA revealed a significant main effect of marker duration $[F(2,24)=$ 5.05] and a significant marker duration $\times$ group interaction $[F(2,24)=3.93]$. One-way ANOVAs performed on the PSEs in each group revealed that the effect of marker duration was reliable only in group filled-first $[F(2,12)=11.09]$.

\section{Discussion}

In both groups, the PSE was close to the geometric mean between the short and the long samples, somewhat under that value in group empty-first and somewhat over that value in group filled-first. Hence, it appears that the same timing system is used for empty and filled intervals, because in both cases the PSE approximates the geometric mean of the short and the long samples, a result frequently cited as consistent with a scalar timing system (e.g., Allan \& Gibbon, 1991; Gibbon, 1986). This is not to say, however, that subjective time necessarily accumulates at the same rate during empty and filled intervals. As Penney, Gibbon, and Meck (2000) have argued, when organisms are trained with a single type of interval (e.g., empty or filled), a scalar timing system should result in a PSE at the geometric mean regardless of the rate at which subjective time accumulates as a function of the passage of real time.

The fact that the PSEs were higher in group filled-first than in group empty-first likely reflects some tendency for pigeons in the former group to time the start, the stop, or both markers. Consistent with this suggestion is the finding that both the percentage of long choices and the PSEs were influenced reliably by marker duration in group filled-first. If the birds in group filled-first began timing at the onset of the start marker and terminated timing when the stop marker terminated, the functional samples during baseline training would be $4 \mathrm{sec}$ (short) and $10 \mathrm{sec}$ (long), because the markers were each $1 \mathrm{sec}$ in duration. Hence, the PSE would be expected to be at the geometric mean of 4 and 10 - that is, to approximate 6.32. Although the calculated PSEs in group filled-first were all considerably below 6.32, the PSEs were calculated using the sample duration only. If the functional sample durations included the marker durations in group filled-first, the calculated PSEs should be increased by the sum of the start and the stop marker durations. Thus, the 4.71 PSE for 0.5-sec markers would become 5.71, the 4.37 PSE for 1-sec markers would become 6.37, and the 3.78 PSE for 2-sec markers would become 7.78. These adjusted PSEs, particularly when the markers were the same duration as in training, approximate expectations based on the notion that the functional samples were 4 and $10 \mathrm{sec}$ in group filled-first. It may be noted that in the psychophysical test that preceded the present test (not reported in this article), the unadjusted PSE in group filled-first was 4.41 with 1-sec start and stop markers, and so the adjusted PSE was 6.41. In our view, these data provide considerable support for the notion that the birds in group filled-first began timing at start marker onset and stopped timing at stop marker termination.

\section{EXPERIMENT 3}

The primary objectives of the present experiment were to (1) make a within-subjects comparison of retention of empty and filled intervals and (2) assess the effect of prior training with one type of interval, either empty or filled, on retention of the other type of interval. To accomplish this, the pigeons in group empty-first also received training with filled intervals, and the pigeons in group filled-first also received training with empty intervals. Following acquisition, retention was assessed on both empty- and filled-interval trials in both groups.

\section{Method}

\section{Subjects and Apparatus}

The subjects were the same as those in Experiment 2. The apparatus was the same as that in Experiment 1.

\section{Procedure}

Training. Sessions were identical for both groups and consisted of 64 trials, 16 with each of the four sample types (short empty, long empty, short filled, and long filled). In both groups, a different set of comparisons was presented following empty and filled sample trials. Hence, those receiving colors in the task trained in Experiment 1 received lines following the samples introduced in this experiment, and vice versa. The position of the correct comparison stimulus was equally often right and left with each sample type in each session. The order of trials varied randomly from session to session and from pigeon to pigeon. All other aspects of the procedure were the same as those in Experiment 1.

All the birds received a minimum of 96 sessions of training. At that point, the subjects that had met a criterion of $85 \%$ correct or higher over two consecutive blocks of 4 sessions on each task were advanced to retention testing. The subjects that had not met criterion after 96 sessions continued on training until either criterion was met and the subject was advanced to testing or the experimenter concluded that the subject would not reach criterion and the subject was dropped from the experiment. Two subjects in each group failed to reach criterion. Thus, there were 5 subjects tested in each group.

Extended-delay testing. Sessions were identical to those in the first test phase in Experiment 1, except that the 64 trials per session were equally divided among the four, rather than two, sample types. In all the test sessions, each of the four samples occurred equally often before each of the three delays $(0.5,2 \pm 1$, and $10 \mathrm{sec})$, and the position of the correct comparison stimulus was balanced within sample type and delay interval factors. Correct responses on extended-delay trials were reinforced in accord with the contingencies of training.

All the subjects received 16 test sessions. Each test session after the first was preceded by 2 baseline sessions identical to those in training.

\section{Results}

\section{Training}

Throughout training, accuracy on the initially trained task remained high and stable for all the subjects. Four subjects from each of the two groups met criterion after 
96 sessions of training and were moved to extendeddelay testing. An additional bird from each group met criterion after 104 sessions of training and was moved to extended-delay testing. The remaining 4 birds, 2 from each group, remained on training for 156 sessions, at which point they were dropped from the experiment because their accuracy on the second trained task remained well below criterion. Hence, $n=5$ in each group.

\section{Extended-Delay Testing}

Data from retention testing are shown in Figure 5. Examining retention on the task trained initially reveals that training on the other set of intervals had little effect on the pattern of retention on the task trained initially. Thus, group empty-first (top panel) demonstrated a robust CLE on empty trials, as was the case in Experiment 1. Similarly, group filled-first (bottom panel) demonstrated little evidence of retention asymmetry on filled-interval trials, although accuracy was somewhat higher on shortthan on long-sample trials at the 10-sec delay. Again, this pattern is identical to that obtained in Experiment 1.

Consider next the data from the task trained second. In group empty-first, prior training with empty intervals appeared to affect retention on filled-interval trials. In
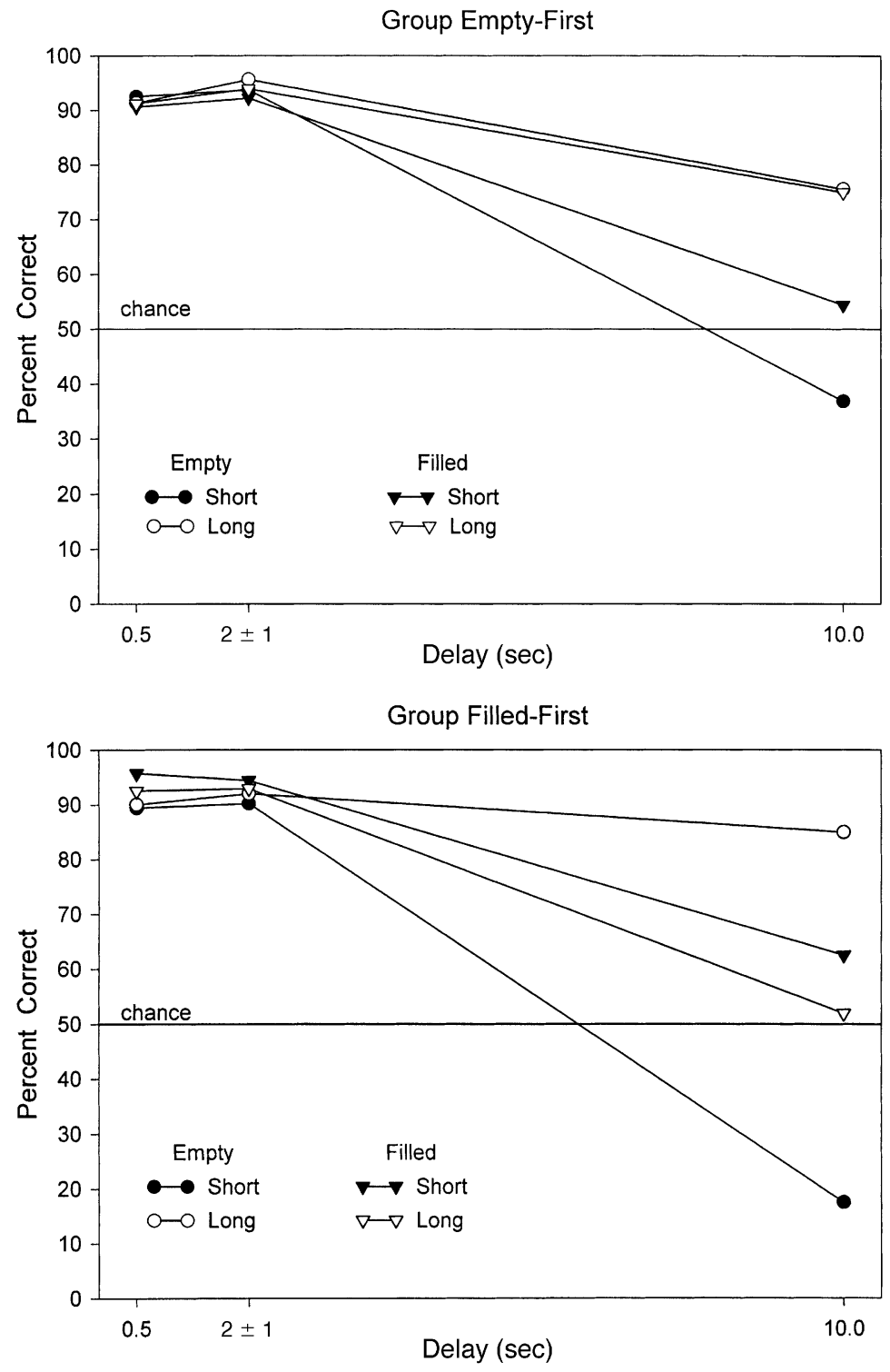

Figure 5. Percentages of correct responses as a function of delay on shortand long-sample trials in group empty-first (top graph) and filled-first (bottom graph) in Experiment 3. 
particular, these subjects demonstrated a modest CLE with filled intervals, rather than the weak CSE shown by group filled-first in this experiment and in Experiment 1. Four of the 5 birds demonstrated a CLE with filled intervals, and the remaining bird demonstrated a modest CSE. On the other hand, initial training on filled intervals appeared to have little effect on retention of empty intervals in group filled-first, since group filled-first demonstrated a robust CLE with empty samples. All 5 birds demonstrated a robust CLE with empty intervals.

A group $\times$ sample type (empty or filled) $\times$ sample duration $\times$ delay ANOVA revealed a significant quadruple interaction $[F(2,16)=3.84]$. Therefore, separate sample type $\times$ sample duration $\times$ delay ANOVAs were performed on the data from each group. The ANOVA on data from group empty-first revealed a significant main effect of delay $[F(2,8)=117.39]$ and a significant sample duration $\times$ delay interaction $[F(2,8)=6.66]$. Separate sample type $\times$ delay ANOVAs confirmed the reliability of the CLE on both empty-interval $[F(2,8)=5.47]$ and filled-interval $[F(2,8)=4.84]$ trials.

The ANOVA on data from group filled-first revealed a significant sample type $\times$ sample duration $\times$ delay interaction $[F(2,8)=9.50]$. This triple interaction indicated that as the delay increased, the increased tendency to choose the comparison associated with either the long or the short sample depended on whether the sample type was empty or filled. To further analyze this interaction, separate sample duration $\times$ delay ANOVAs were conducted for empty and filled trials. The analysis for the empty trials showed a significant sample duration $\times$ delay interaction $[F(2,8)=46.37]$, which indicated a significant CLE. In contrast, the analysis for the filled trials showed no significant main effects or interactions, which indicated a nonsignificant CSE.

\section{Discussion}

The finding of a CLE with filled intervals in group empty-first suggests that prior training with empty intervals alters the way pigeons code filled intervals. Had that not been the case, either a robust CSE, as in previous studies with filled intervals cited earlier in this article, or a weak CSE, as in group filled-first in the present experiments, would have been expected. However, the precise way in which the processing of filled intervals was altered is not immediately obvious.

In contrast to group empty-first, group filled-first provided no evidence that prior training with filled intervals alters the way pigeons process empty intervals. In particular, a robust CLE was obtained on emptyinterval trials in group filled-first, as was the case in group empty-first in Experiment 1 and in groups tested with empty intervals in prior studies (Grant, 2001; Santi et al., 2003; Santi et al., 1999).

\section{EXPERIMENT 4}

In the present experiment, the psychophysical test reported as Experiment 2 in the present series was re- peated, except that both groups were tested with both empty and filled intervals. Recall that in Experiment 2, there was evidence that group filled-first timed the start and stop markers on filled-sample trials, whereas there was no evidence that group empty-first timed the start and stop markers on empty-interval trials. Interest focused on whether both groups might now demonstrate that same pattern - that is, timing the start and stop markers only on filled-interval trials.

\section{Method}

\section{Subjects and Apparatus}

The 10 subjects that met criterion in Experiment 3 were employed. The apparatus was the same as that in Experiment 1.

\section{Procedure}

The six types of probe sessions ( 2 interval types [empty or filled] $\times 3$ marker durations $[0.5,1$, or $2 \mathrm{sec}])$ were identical to those in Experiment 2. In the present experiment, both groups were tested on each of the six types of probe sessions. The experiment consisted of 96 consecutive probe sessions, 16 with each of the six types of probe sessions. For the pigeons in each group, sessions with empty and filled intervals alternated, and the marker duration was determined randomly, with the constraint that each of the three marker durations was presented with each interval type once every 6 sessions. The PSE was determined using the procedure specified in Experiment 2.

\section{Results}

Figure 6 shows the percentage of long choices as a function of sample duration on probe trials, with marker duration as the parameter. Data from group empty-first are shown in the top graphs, and those from group filledfirst are shown in the lower graphs. Data from emptyinterval trials are shown in the left graphs, and data from filled-interval trials are shown in the right graphs.

The data from the present within-subjects test are highly similar to those obtained in the between-subjects test. Specifically, there was little evidence that the pigeons in either group timed the markers on emptyinterval trials. On filled-interval trials, on the other hand, the percentage of long choices increased as marker duration increased, suggesting that the pigeons in both groups timed the markers on filled-interval trials.

A group $\times$ sample type $\times$ sample duration $\times$ marker duration ANOVA was performed on the data from probe trials. Because the ANOVA revealed a significant sample type $\times$ sample duration $\times$ marker duration interaction $[F(12,96)=2.80]$, separate group $\times$ sample duration $\times$ marker duration ANOVAs were performed on the data from empty- and filled-interval trials. The ANOVA on empty-interval trials revealed a significant main effect of sample duration $[F(6,48)=133.04]$ as the only significant term. The ANOVA on filled-interval trials revealed significant main effects of sample duration $[F(6,48)=94.29]$ and marker duration $[F(2,16)=14.48]$. In addition, the ANOVA revealed a significant sample duration $\times$ marker duration interaction $[F(12,96)=4.61]$. The interaction likely resulted from a ceiling effect at longer sample durations that partially masked the effect of marker duration. 

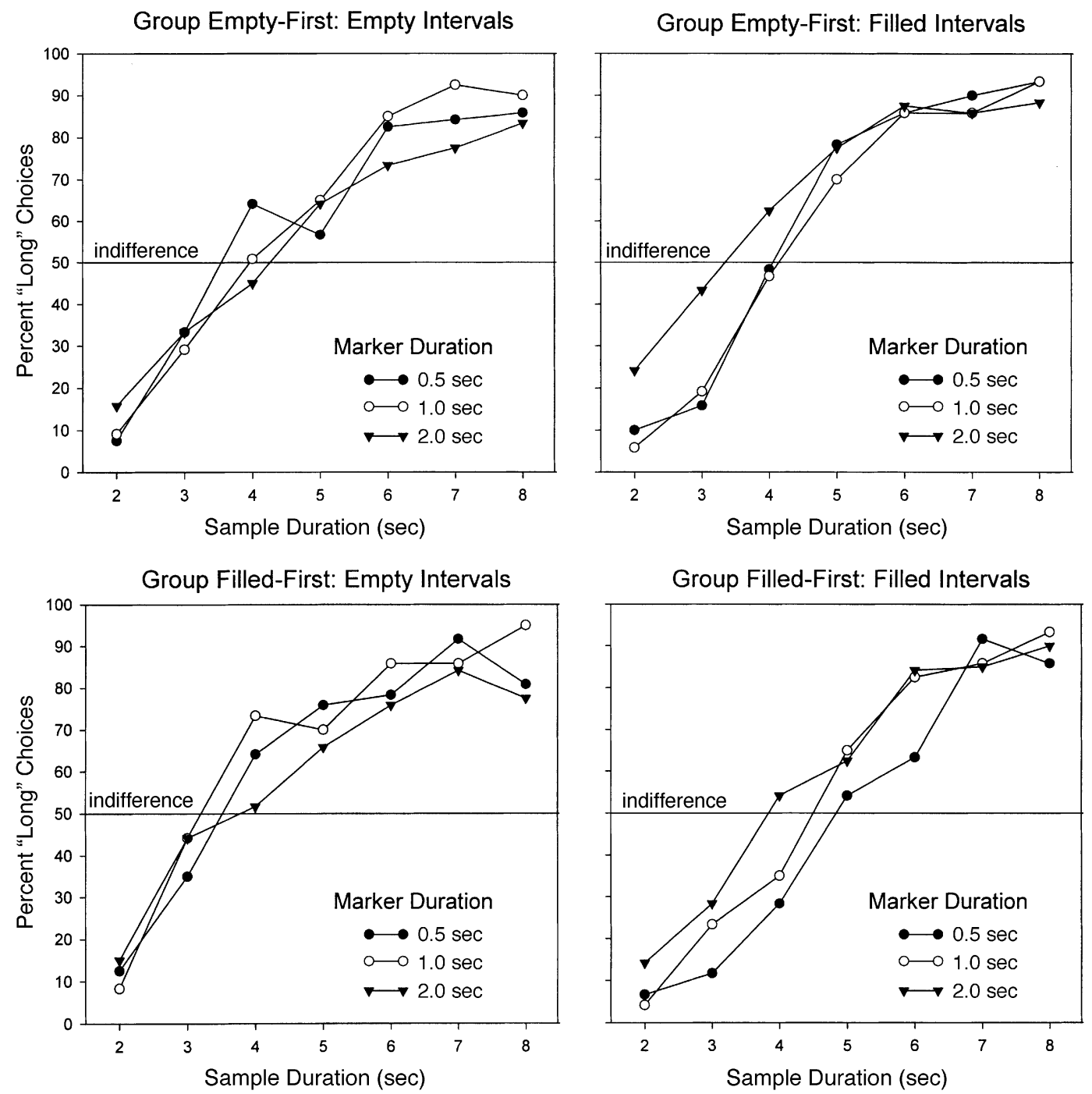

Figure 6. Percentages of choices of the long-associated comparison stimulus as a function of sample stimulus duration in group empty-first (top graphs) and filled-first (bottom graphs) in Experiment 4. Marker duration (i.e., duration of the start and stop signals) is the parameter. Data from empty-interval trials are shown in the two leftmost graphs, and data from filled-interval trials are shown in the two rightmost graphs.

The PSEs on empty-interval trials revealed no systematic effect of marker duration in either group. On filled-interval trials, however, the PSE decreased monotonically as marker duration increased. A group $\times$ sample type $\times$ marker duration ANOVA performed on the PSEs revealed a significant sample type $\times$ marker duration interaction $[F(2,16)=21.51]$. To further assess the interaction, separate group $\times$ marker duration ANOVAs were performed on the data from empty- and filled-interval sessions. The analysis on empty-interval data revealed no significant terms, and the analysis on filled-interval data revealed a main effect of marker duration $[F(2,16)=12.05]$ as the only significant term. Because there were no significant terms involving the group factor in any analysis, a single mean PSE was calculated for each marker duration with each sample type.
Mean PSEs at the 0.5-, 1-, and 2-sec marker durations were $3.95,3.88$, and 4.11, respectively, on the empty-interval trials and were $4.61,4.31$, and 3.76, respectively, on the filled-interval trials.

\section{Discussion}

In Experiment 2, the pigeons trained and tested with filled intervals tended to time the markers, whereas those trained and tested with empty intervals did not. In the present experiment, both groups were tested with empty and filled intervals. On filled-interval trials, the percentage of long choices increased and the PSE decreased as marker duration increased. Both of these findings suggest that the pigeons tended to time the markers and, moreover, to add time spent in the presence of the marker to time spent in the presence of the nominal sample. On 
empty trials, on the other hand, there was no systematic relation between marker duration and either percentage of long choices or PSE, suggesting that the markers on empty-interval trials were not timed.

It should be noted that although the pigeons in both groups provided evidence that the markers on filledinterval trials were timed, the retention functions produced on filled-interval trials were different. Experiment 3 revealed a strong CLE on filled-interval trials in group empty-first and a weak CSE on these trials in group filled-first. Moreover, group empty-first also demonstrated a strong CLE on empty-interval trials, even though the markers were apparently not timed on empty-interval trials. Overall, therefore, there is little evidence that whether the markers are or are not timed is solely responsible for either the type of retention asymmetry (i.e., CLE or CSE) or the strength of retention asymmetries.

It may seem curious that markers would be timed on filled-interval, but not on empty-interval, trials, particularly since filled and empty samples were intermixed randomly during training sessions. Hence, it was not until termination of the start marker that a bird could discriminate an empty-interval from a filled-interval trial. It seems likely, therefore, that the birds had an equivalent tendency to time the start marker on both empty- and filled-interval trials. Because marker duration did not influence performance on empty-interval trials, it appears that onset of the empty interval following termination of the start marker reset the timing mechanism. Alternatively, perhaps it was only the stop marker that was timed, but only when it was preceded by a filled interval.

\section{GENERAL DISCUSSION}

The series of experiments reported in this article extend our knowledge concerning the processing of temporal intervals in a number of ways. First, the CLE obtained with empty intervals does not appear to arise exclusively from the presentation of start and stop markers. Although presentation of start and stop markers on filled-interval trials eliminated the CSE typically obtained, their presentation did not result in a CLE. Second, prior training with empty intervals alters the way in which pigeons process, code, and/or retain information from the presentation of filled intervals. In particular, prior training with empty intervals results in a CLE, rather than the typical CSE with filled intervals. Third, prior training with filled intervals does not influence the processing, coding, and/or retention of information from the presentation of empty intervals. In particular, group filled-first demonstrated a robust CLE with empty intervals, as is the case in the absence of prior training with filled intervals (group empty-first in Experiment 1; Grant, 2001; Santi et al., 2003; Santi et al., 1999). Fourth, the PSE on empty trials approximates the geometric mean of the short (2-sec) and the long (8-sec) samples, as is typically the case with filled intervals (e.g., Gibbon, 1986; Miki \& Santi, 2001; Spetch \& Wilkie, 1983; Stubbs,
1968). Hence, although retention is strongly affected by whether an interval is empty or filled, the mechanisms of timing that interval appear not to be so affected. Finally, pigeons have a tendency to time the markers and add that time to the time spent in the presence of the nominal sample on filled-interval, but not on empty-interval, trials.

Prior research has ruled out several potential causes of the CLE obtained when short and long empty intervals are used as samples. For example, Grant (2001) showed that when different stimuli consistently served as start and stop markers, as was the case in the experiments reported in the present article, presentation of the stop marker did not initiate the timing of a new interval. In addition, Santi et al. (2003) showed that the CLE did not arise from (1) either counting or timing the markers or (2) asymmetrical coding and default responding.

Santi et al. (2003) suggested that perhaps the detection model of Gaitan and Wixted (2000) might provide a viable account of the CLE with empty intervals. Gaitan and Wixted maintained that whenever samples differ markedly in salience, pigeons attempt to retrieve the memory for the more salient sample at testing, responding to the alternate comparison by default in the absence of memory for the more salient sample. On this view, the CSE typically obtained with filled samples arises because the long sample is more salient and, therefore, presentation of the comparisons results in a search of memory for the long sample. When the long sample memory is not retrieved, either because the long sample was not presented or because the presentation of a long sample had been forgotten, the pigeon chooses the comparison associated with the short sample. Santi et al. (2003) noted that Gaitan and Wixted's detection model would predict a CLE with empty intervals, provided it is assumed that the short empty sample is more salient than the long empty sample. Such an assumption is plausible considering that short empty samples involve two light presentations (i.e., the start and stop markers) in a relatively short time interval, whereas long empty samples involve two light presentations over a much more temporally extended period.

However, the data from Experiment 3 would appear particularly challenging to the detection model. In that experiment, group empty-first demonstrated a CLE on both empty and filled trials. Therefore, the detection model would need to argue that, in this case, it is the short-filled, rather than the long-filled, sample that is more salient. One could argue, perhaps, that pigeons in group empty-first evaluated the salience of filled intervals not in terms of duration, but in terms of the number of different stimuli presented per unit of time. Because markers were presented with filled intervals in the present experiments, filled samples involved three stimulus presentations, and those presentations occurred across a relatively short interval on short-sample trials and over a relatively long interval on long-sample trials. However, the psychophysical data reported in Experiments 2 and 4 strongly suggest that the birds in both groups were indeed timing both empty and filled intervals and that du- 
ration controlled choice responding. In our view, it is unlikely that the detection model can provide a coherent explanation of all the present findings.

Alternatively, it may be that the CLE arises when empty intervals of different duration are used as samples because pigeons time during the delay on at least some proportion of trials. On this view, pigeons would tend to choose the long-associated comparison stimulus after a long delay because, on at least some proportion of trials, the timing process continued during the long delay and, therefore, the result of the timing process would be judged as more similar to a long than to a short sample. A variable delay was used during baseline training, both in the experiments reported in this article and in those reported by Grant (2001), to render the sample-plusdelay duration a less discriminable cue for choice than was the sample duration alone. It was anticipated, therefore, that pigeons would learn to use the stop marker, rather than the onset of the comparisons, as the signal to stop timing. Nonetheless, it is possible, although unlikely, as will be argued later in this discussion, that the comparisons, rather than the stop marker, terminated timing for at least some pigeons on some proportion of trials.

Indeed, Dorrance et al. (2000) found evidence that the comparisons, rather than sample offset, can serve as the cue to stop timing under some conditions in a filledinterval procedure. Specifically, when the onset of a 2- or 10 -sec keylight sample was accompanied by termination of a houselight that had been illuminated throughout the ITI, pigeons demonstrated a CLE at longer testing delays. This occurred, moreover, despite the fact that a delay of $0,1,2$, and 4 sec occurred on training trials. Dorrance et al. suggested that termination of the houselight was a more salient event than was onset of the keylight sample and, hence, was the event that initiated the timing process. They suggested further that, perhaps because onset of the keylight did not initiate timing, offset of the keylight did not terminate timing and, instead, the onset of the comparisons, a highly salient event, served to terminate the timing process.

In the present experiments, however, we believe that it is unlikely that onset of the comparisons, rather than onset of the stop marker, terminated the timing process. In particular, the stop marker consisted of three illuminated keylights, whereas the comparisons consisted of only two illuminated keylights. Thus, onset of the stop marker was likely no less salient than onset of the comparisons. Moreover, Experiments 2 and 4 revealed that pigeons do not time the stop marker on empty-interval trials in the present procedure. It seems implausible to argue that pigeons timed during the empty interval (i.e., the sample) stopped timing during the stop marker and then restarted timing during the delay, particularly so given Grant's (2001) finding that presentation of the second marker did not initiate timing a new interval when, as in the present experiments, Stimulus A always served as the start marker and Stimulus B always served as the stop marker. Given that (1) the second marker was not timed (based on Experiments 2 and 4) and (2) the second marker did not initiate timing a new interval (based on Grant, 2001), it appears unlikely that the birds were timing during the delay interval on empty-interval trials. Additional research is clearly required to identify the mechanism(s) responsible for the CLE with empty intervals.

Finally, some consideration should be given to the fact that a statistically reliable CSE was not obtained in group filled-first in Experiment 1 at a point in the training regime at which this group had experienced only filled intervals. It may be that the use of markers altered the timing task in some fundamental way. For example, the pigeons may have coded the number of recent stimulus changes perceived (e.g., three on short trials and two on long trials) or, perhaps, coded the samples in terms of rate of stimulus change (high on short trials and low on long trials). Alternatively, it may be that the introduction of start and stop markers caused the pigeons to code the filled interval that separated the two markers categorically as short or long (or perhaps prospectively-for example, peck red or peck green). On this view, forgetting of the code would result in random responding and, hence, unbiased retention functions. It should be admitted, however, that we are unable to suggest a compelling reason why use of markers would have such an effect.

Another possible explanation for the lack of a CSE on filled-interval trials in group filled-first is suggested by the results of psychophysical testing in Experiments 2 and 4 . In those experiments, performance on filledinterval trials in group filled-first was influenced by the duration of the markers, so that a higher proportion of long choices and lower PSEs was obtained with longer markers. This result suggests that the pigeons in this group timed the filled samples from the onset of the start marker to the termination of the stop marker. If so, the functional samples on filled-interval trials may have been closer to 4 and $10 \mathrm{sec}$ than to the nominal values of 2 and $8 \mathrm{sec}$. Consistent with this proposition is the finding that the adjusted PSE was very close to the geometric mean of 4 and 10, particularly when the markers on probe trials were the same duration as those on baseline trials.

To the best of our knowledge, no experiment has been reported using 4- and 10-sec filled intervals. In most experiments, the ratio of short sample duration to long sample duration has been 1:4 or 1:5. Nonetheless, it is not obvious why, from a subjective shortening perspective, a CSE would not be obtained with 4- and 10 -sec samples. From the perspective of a detection model, it could be argued, perhaps, that 4 and $10 \mathrm{sec}$ do not differ sufficiently in salience to evoke the selective retrieval process. It may be noted, however, that Grant and Kelly (1996, Experiment 2) obtained a robust CSE using 2(short) and 3-sec (long) samples. Hence, it would appear that a small short-to-long ratio does not, in itself, preclude the occurrence of a strong CSE.

An experiment we are currently conducting might clarify the issue of why a CSE was not obtained with filled intervals in group filled-first. One group will receive 2- and 
8-sec samples, and another will receive 4- and 10-sec samples; neither group will receive markers. A final group will receive 2 - and 8 -sec samples with 1 -sec start and stop markers. If the markers alter the way samples are coded, only the marker group should fail to demonstrate a CSE. If, on the other hand, a CSE does not occur with 4- and 10 -sec samples, only the group receiving 2 - and 8 -sec samples without markers should demonstrate a CSE.

Finally, it should be noted that the terms empty interval and filled interval have been used throughout this article. It is the case, of course, that both the empty and the filled intervals employed were marked by brief start and stop markers. Markers were used on filled-interval trials so that empty- and filled-interval samples would differ only in whether the duration was empty or filled. However, it is possible that the conclusions supported by the research reported in this article might not generalize to filled-interval samples that are neither preceded nor followed by presentation of a marker. Additional research on this issue is warranted.

\section{REFERENCES}

Allan, L. G., \& GibBon, J. (1991). Human bisection at the geometric mean. Learning \& Motivation, 22, 39-58.

Dorrance, B. R., Kaiser, D. H., \& Zentall, T. R. (2000). Eventduration discrimination by pigeons: The choose-short effect may result from retention-test novelty. Animal Learning \& Behavior, 28, 344-353.

Gaitan, S. C., \& Wixted, J. T. (2000). The role of "nothing" in memory for event duration in pigeons. Animal Learning \& Behavior, 28, 147-161.

GibBon, J. (1986). The structure of subjective time: How time flies. In G. Bower (Ed.), The psychology of learning and motivation (Vol. 20, pp. 105-135). New York: Academic Press.

Grant, D. S. (1993). Coding processes in pigeons. In T. R. Zentall (Ed.), Animal cognition: A tribute to Donald A. Riley (pp. 193-216). Hillsdale, NJ: Erlbaum.

GRANT, D. S. (2001). Memory for empty time intervals in pigeons. $A n$ imal Learning \& Behavior, 29, 293-301.

GrANT, D. S., \& KelLy, R. (1996). The role of minimum wait time and sample discriminability in the coding of event duration in pigeons. Learning \& Motivation, 27, 243-259.

GRANT, D. S., \& KelLY, R. (1998). The effect of variable-delay training on coding of event duration in pigeons. Learning \& Motivation, 29, 49-67.

GRANT, D. S., \& KeLLy, R. (2001). Many-to-one matching with temporal and hedonic samples in pigeons. Learning \& Motivation, $\underline{32}_{2}$ 477-498.

GrANT, D. S., \& Spetch, M. L. (1991). Pigeons' memory for event duration: Differences between choice and successive matching tasks. Learning \& Motivation, 22, 180-199.

GrANT, D. S., \& SPETCH, M. L. (1993). Analogical and nonanalogical coding of samples differing in duration in a choice-matching task in pigeons. Journal of Experimental Psychology: Animal Behavior Processes, $\mathbf{1 9}, 15-25$.
Grant, D. S., \& SPETCH, M. L. (1994). The role of asymmetrical coding of duration samples in producing the choose-short effect in pigeons. Learning \& Motivation, 25, 413-430.

Grant, D. S., Spetch, M. L., \& KELLY, R. (1997). Pigeons' coding of event duration in delayed matching-to-sample. In C. M. Bradshaw \& E. Szabadi (Eds.), Time and behaviour: Psychological and neurobehavioural analyses (pp. 217-264). Amsterdam: Elsevier.

Kraemer, P. J., Mazmanian, D. S., \& Roberts, W. A. (1985). The choose-short effect in pigeon memory for stimulus duration: Subjective shortening versus coding models. Animal Learning \& Behavior, 13, 349-354.

MIKI, A., \& SANTI, A. (2001). Pigeons' timing of an arbitrary and a naturalistic auditory stimulus: Tone versus cooing. Behavioural Processes, 53, 103-111.

Penney, T. B., GibBon, J., \& Meck, W. H. (2000). Differential effects of auditory and visual signals on clock speed and temporal memory. Journal of Experimental Psychology: Human Perception \& Performance, 26, 1770-1787.

RoBERTS, W. A., MACUDA, T., \& BRoDBECK, D. R. (1995). Memory for number of light flashes in the pigeon. Animal Learning \& Behavior, 23, $182-188$.

SaNTI, A., BRIDSon, S., \& Ducharme, M. J. (1993). Memory codes for temporal and nontemporal samples in many-to-one matching by pigeons. Animal Learning \& Behavior, 21, 120-130.

Santi, A., Hornyak, S., \& Miki, A. (2003). Pigeons' memory for empty and filled time intervals signaled by light. Learning \& Motivation, 34, 282-302.

Santi, A., Ross, L., Coppa, R., \& Coyle, J. (1999). Pigeons' memory for empty time intervals marked by visual or auditory stimuli. Animal Learning \& Behavior, 27, 190-205.

SPETCH, M. L. (1987). Systematic errors in pigeons' memory for event duration: Interaction between training and test delay. Animal Learning \& Behavior, 15, 1-5.

Spetch, M. L., Grant, D. S., \& Kelly, R. (1996). Procedural determinants of coding processes in pigeons' memory for duration. Learning \& Motivation, 27, 179-199.

Spetch, M. L., \& RUSAK, B. (1992a). Temporal context effects in pigeons' memory for event duration. Learning \& Motivation, 23, 117 144.

SpeTCH, M. L., \& RusaK, B. (1992b). "Time present and time past.” In W. K. Honig \& J. G. Fetterman (Eds.), Cognitive aspects of stimulus control (pp. 47-67). Hillsdale, NJ: Erlbaum.

SPETCH, M. L., \& WiLKIE, D. M. (1982). A systematic bias in pigeons' memory for food and light durations. Behavior Analysis Letters, 2 , 267-274.

Spetch, M. L., \& WiLKie, D. M. (1983). Subjective shortening: A model of pigeons' memory for event duration. Journal of Experimental Psychology: Animal Behavior Processes, 9, 14-30.

STUBBS, D. A. (1968). The discrimination of stimulus duration by pigeons. Journal of the Experimental Analysis of Behavior, 11, 223238

WILKIE, D. M. (1987). Stimulus intensity affects pigeons' timing behavior: Implications for an internal clock model. Animal Learning \& Behavior, 15, 35-39.

(Manuscript received November 17, 2003, revision accepted for publication April 9, 2004.) 